\title{
PQLI Design Space
}

\author{
John Lepore • James Spavins
}

Published online: 17 May 2008

(C) International Society for Pharmaceutical Engineering 2008

\begin{abstract}
This paper describes progress made by the Design Space Task Team within the ISPE Product Quality Lifecycle Implementation (PQLI) initiative. It is intended to provide approaches to the rational development of Design Space, as well as background on Design Space, its historical origins and how it fits within the wider PQLI initiative. The focus of this paper is on the technical elements of Design Space development.
\end{abstract}

Keywords PQLI · Design space $\cdot$ Criticality $\cdot$ Control strategy $\cdot$ Critical quality attribute $\cdot$ Critical process parameter $\cdot$ Knowledge space $\cdot$ PAT $\cdot$ Pharmaceutical target product profile $\cdot$ Product and process knowledge $\cdot$ Quality by design - Quality risk management

\section{Introduction}

This paper outlines the steps and elements for determining a Design Space for pharmaceutical products and how this Design Space can be used by industry to assure quality products, patient safety and product efficacy.

Contributing Team Members: Theodora Kourti - GSK, Alastair Coupe - Pfizer, Vincent McCurdy - Pfizer, Richard Saunders - Wyeth, Kim Vukovinsky - Pfizer, William Spanogle - J\&J, Steve

Laurenz - Abbott, Kevin Seibert - Lilly, Paul Stott - AstraZeneca,

Tim Watson - Pfizer

\author{
J. Lepore \\ Merck \& Co., Inc., \\ 126 E. Lincoln Ave., Rahway, NJ 07065, USA \\ e-mail: lepore@merck.com \\ J. Spavins \\ Pfizer Inc., \\ 1 Eastern Point Rd., Groton, CT 06340, USA \\ e-mail: james.c.spavins@pfizer.com
}

The general engineering and technical design processes discussed here have been widely used by many types of industries in addition to the pharmaceutical industry (including aerospace, food, computer, civil and mechanical engineering and medical devices) and the use of risk based analyses to determine design constraints and then determine appropriate controls is a foundational process to the advancement of science and technology. Accordingly, it is appropriate for the pharmaceutical industry to expand the use of these practices.

The paper seeks to clearly define what Design Space is, and provide some guidance as to how to achieve it. It also discusses issues including the relationship with Proven Acceptable Ranges (PARs).

ICH Q8 [1] defines Design Space as:

"...the multidimensional combination and interaction of input variables (e.g., material attributes) and process parameters that have been demonstrated to provide assurance of quality"

There are many scientifically justifiable means towards achieving a Design Space. The approach to any specific project can harness any combination of tools, depending on the specific technologies being evaluated, available literature, internal corporate experience, and comfort level.

Design Space, especially when linked with a structured Control Strategy, and criticality assessment, has the potential to change aspects of regulatory interactions, providing data and context while continuing to provide a high level of assurance for pharmaceutical quality and performance.

Use of the Design Space approach allows more effective dialogue between industry and regulator during the application review process. It allows the regulator to more readily see the connection between Design Space, criticality and Control Strategy. It serves industry by 
providing a structured and deliberate approach that is well aligned with a risk and science based approach to product development.

There will be a wide range of approaches to the analysis and output for a specific product, but transparency and clarity is necessary, to explain and justify the approach, and provide the foundation for continuous improvement. Considerations on how to effectively present the Design Space are given later in this paper.

If performed and articulated well, Design Space together with an appropriate Control Strategy will reduce and focus end product testing, while increasing process performance and robustness. There are inherent benefits for taking this approach, although it should be noted that traditional approaches continue to be completely acceptable.

Development resources may be required to achieve a knowledge rich understanding of the process, but this investment can be offset by certitude of scale-up, more consistent manufacturing, and potential regulatory flexibility.

This paper describes progress made by the Design Space Task Team within the ISPE PQLI initiative - a global industryled three to five year initiative aimed at facilitating the implementation of ICH Q8, Q9 [3], and ultimately Q10 [4] guidance.

This should be regarded as work in progress and comment and input from industry, regulators, and other stakeholders is welcomed.

\section{Developing a Design Space}

Design Space is dynamic and begins at drug conceptualization and continues to evolve over the entire lifecycle of the process. At the time of initial product commercialization, the Design Space can be considered to represent the best overall process understanding at the time. It continues to evolve, not because of lack of initial development effort, but as additional knowledge and information is generated throughout the lifecycle of the product: a key element of continuous improvement programs.
Design space is determined by an iterative process. The process is not complete until successive iterations demonstrate appropriate understanding of attributes needed to assure the Pharmaceutical Target Product Profile. While this may vary for specific product, for many pharmaceutical presentations the profile needed will be widely understood, at least at a high level. Understanding of clinical needs and therapeutic index are important, for example, in determining this profile. Fig. 1.

Design Space is linked to criticality through the results of risk assessment, which differentiates between those product attributes and process parameters that are critical to product quality (with underlying assumption of patient safety and efficacy, and determining Critical Quality Attributes (CQAs) and Critical Process Parameters (CPPs)).

ICH Q9 provides an excellent framework for Quality Risk Management, including risk assessment. It also refers to existing tools such as HACCP (Hazard Analysis and Critical Control Points), 'Worst Outcomes Analysis', FMECA (Failure Mode Effects and Criticality Analysis) [5], any of which can provide a framework or be adapted to provide a process for risk assessment.

Design Space may include information regarding site, equipment and scale of manufacture, depending on the level of the process knowledge, and the sensitivity of process performance on those aspects.

A representation of Design Space and its links to overall product and process knowledge and normal operating ranges is given in Fig. 2.

Critical Quality Attributes and Critical Process Parameters, once identified and understood, will need to be controlled. Suitable systems and management processes can ensure disciplined implementation of and adherence to the design intent of the process. The forthcoming ICH Q10, which addresses Pharmaceutical Quality Management Systems, will provide guidance on these aspects. This outlines expectations for the Pharmaceutical Quality Systems, and how they can be applied in the management of the Design Space, risk assessment, and to ensure that quality standards are met over the lifecycle of the product.

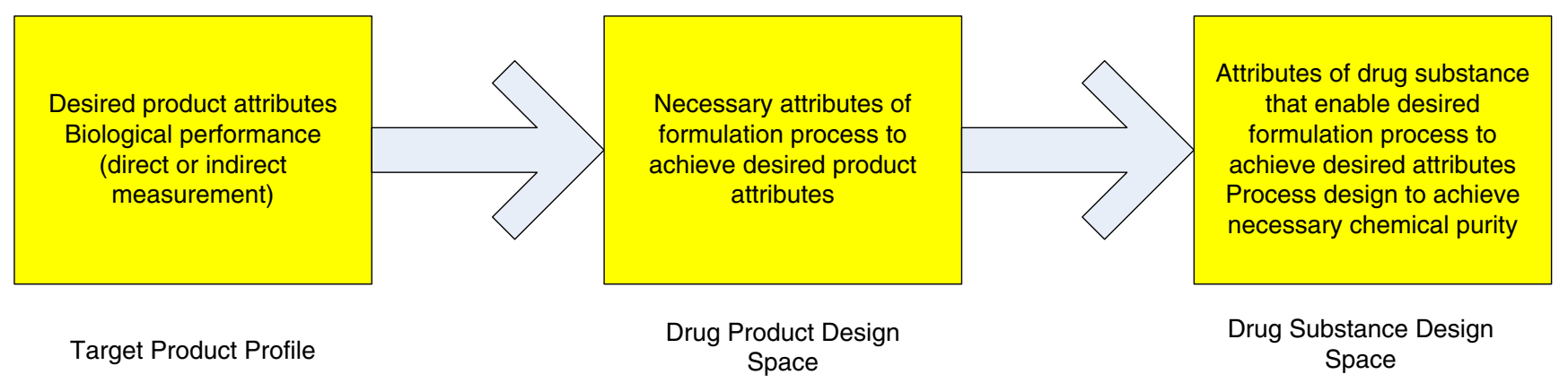

Fig. 1 Developing a design space 
Fig. 2 Representation of design space

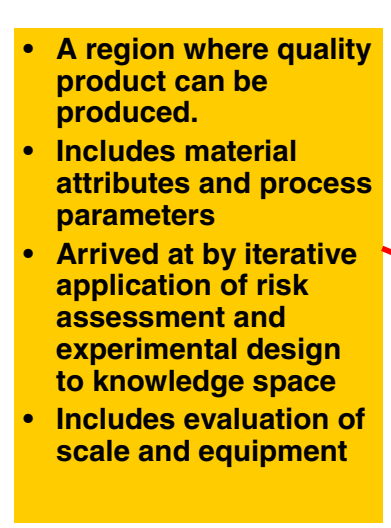

Procedure for Determining a Design Space

Design Space is initially determined during product development through an iterative process. Initially, the links of the process to the profile may be tenuous, as central elements of safety margins, clinical dose range, and the like are becoming understood in parallel with the early conceptualization and development of the process. It is not unreasonable, therefore, to take a phased approach, taking advantage of new safety and clinical information to enhance the strength of process-product linkages over time.

Examples of quality attributes related to clinical relevance include:

- Biopharmaceutics Classification System (BCS) class

- Physical (stable polymorph) and chemical stability

- Dose level and impacts on processes

- Risks based on formulation and API unit operations

- Particle Size spec established in supportive of product profile
The process flow for a Design Space determination within the overall product and process development is shown in Fig. 3. It is adapted from work by the EFPIA PAT Working Group [6].

Using the Pharmaceutical Product Profile and prior knowledge, a risk assessment is performed. The risk assessment will result in identification of areas where there is a confirmed risk and a need to mitigate or control, or where additional work is needed for further understanding if risk is present.

The ability to determine levels of risk will depend highly on the ability to judge severity and probability of impact. A risk assessment, therefore, is a disciplined and structured approach that clearly shows what has been considered and the outcome of those considerations, including, where necessary, the need to do further work.

The Design Space is linked to criticality through the results of a risk assessment, which determines those critical quality attributes and critical process parameters that must be included in the resultant Design Space. The Design
Fig. 3 Design space in the flow of development

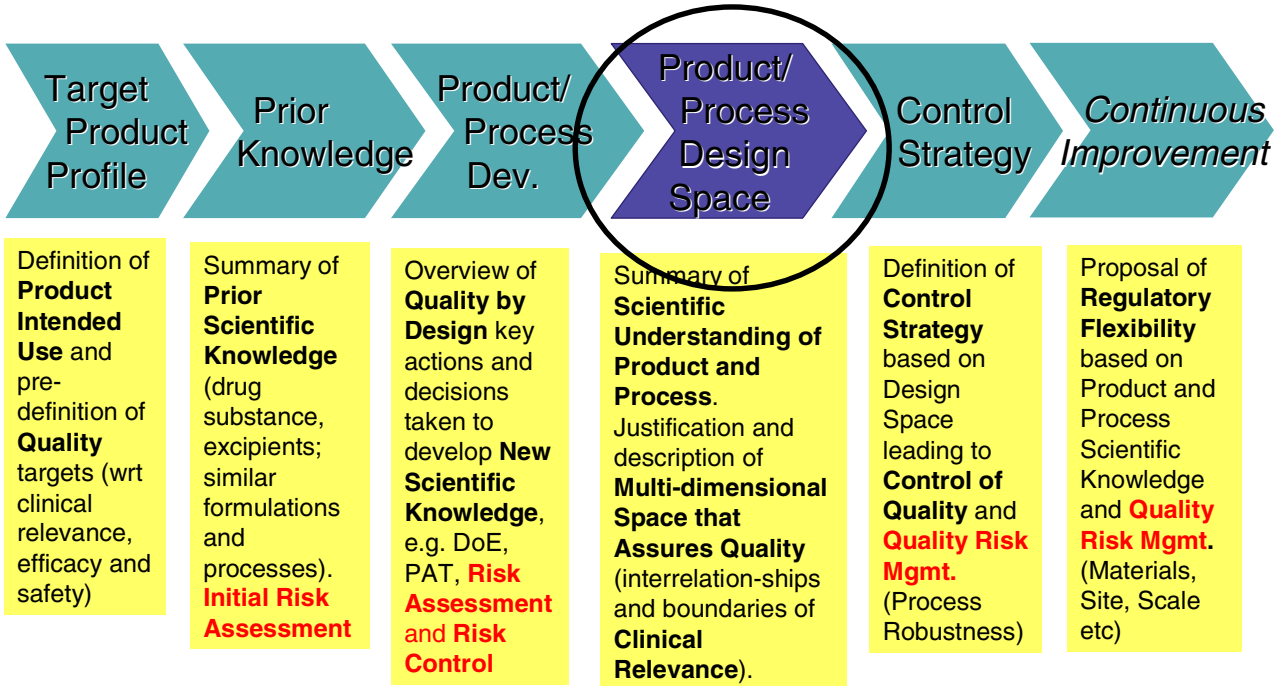

- Adapted from EFPIA PAT Working Group 
Space and the process by which it is arrived at inform decisions on criticality, links functional relationships between Critical Quality Attributes (CQAs) and the Critical Process Parameters (CPPs) that impact them, and should include their linkage across unit operations. Proven acceptable ranges (PARs) are embedded within the design space. It is not necessary to include normal operating ranges in the design space, as they have no impact on a product's Pharmaceutical Target Product Profile.

The Design Space may also contain operating ranges for process parameters classified in an intermediate criticality category used by some companies, and proposed for further consideration by the paper Criticality in PQLI [7] in this Volume of Journal of Pharmaceutical Innovation. Examples may include parameters that are well controlled in relation to the required boundaries and are of lower risk.

The example shows what was considered, and how each attribute was ranked and scored (see Fig. 4). This in turn, informed the experimental strategy that was needed to provide the necessary understanding. Figure 5 shows a nonquantitative example of risk assessment.

\section{Tools for Developing Design Space}

The selection of the correct tools for development of the Design Space may be influenced by the maturity of the underlying science. Where the underlying scientific principles are well understood and developed, it is possible to characterize a process by use of first principle, or mechanistic models. Where such underlying scientific understanding is not possible, scale-up correlations or empirical assessments may be suitable.

There is no single tool or approach that is ideal for every case, and there are specific considerations that can help decide the best tool to use. The principles, assumptions or axioms below may be applied to small and large molecules alike, and to drug substance as well as formulation, but the ability to use them may be limited by knowledge of underlying scientific principles.

Axiom \#1 If first principle relationships prove to be adequately predictive of process performance, then use them. Consequently, there is often an inherent benefit in terms of physical significance.

The places in which first principles are typically encountered are in the thermodynamic and rate phenomena typically associated with chemical processing (with analogous applications in other areas). For example, in chemical reactions, it is often possible to determine kinetic order and rate constant. With appropriate understanding, it is possible to prospectively set reaction conditions to optimize desired outcome.

Similar concepts can apply in thermodynamics, especially in separations, where understanding of phase equilibria and operation within a thermodynamically controlled envelope are important to achieving the intent of the process. In order to fully capitalize on the above first principle knowledge, an understanding of heat and mass transfer effects can provide direction where there may be sensitiv-

Fig. 4 Example risk assessment

\section{Drug Product Risk Assessment - 'Cause \& Effect Matrix' Dry Granulate + Blend Example}

\begin{tabular}{|l|c|c|c|c|c|c|c|c|}
\hline Key Attribute & $\mathbf{Y}$ & $\mathbf{Y}$ & $\mathbf{Y}$ & $\mathbf{Y}$ & $\mathbf{Y}$ & $\mathbf{Y}$ & & \\
\hline Rank & $\mathbf{7}$ & $\mathbf{7}$ & $\mathbf{7}$ & $\mathbf{7}$ & $\mathbf{1 0}$ & $\mathbf{1 0}$ & & \\
\hline Parameter & $\begin{array}{c}\text { Sieve Cut } \\
\text { Potency }\end{array}$ & $\begin{array}{c}\text { Blend } \\
\text { Uniformity }\end{array}$ & $\begin{array}{c}\text { Particle Size } \\
\text { Distribution }\end{array}$ & Mill choking & $\begin{array}{c}\text { Tablet } \\
\text { Hardness }\end{array}$ & $\begin{array}{c}\text { Tablet } \\
\text { Content } \\
\text { Uniformity }\end{array}$ & $\begin{array}{c}\text { Score } \\
\text { Exp. } \\
\text { Strategy }\end{array}$ \\
\hline $\begin{array}{l}\text { Operator Training } \\
\text { Procedures }\end{array}$ & 10 & 10 & 10 & 10 & 10 & 10 & 840 & FMEA \\
\hline Roll Force & 10 & 10 & 10 & 1 & 10 & 10 & 777 & DOE \\
\hline Screen Size & 10 & 10 & 10 & 10 & 5 & 5 & 632 & DOE \\
\hline Gap Width & 10 & 10 & 5 & 5 & 5 & 5 & 585 & DOE \\
\hline Material Throughput & 10 & 1 & 5 & 10 & 1 & 1 & 437 & DOE \\
\hline $\begin{array}{l}\text { Roller Compaction } \\
\text { Calibration }\end{array}$ & 5 & 5 & 5 & 1 & 5 & 5 & 427 & FMEA \\
\hline Sampling Size & 10 & 10 & 10 & 1 & 1 & 5 & 421 & MSA \\
\hline Roll Speed & 5 & 5 & 5 & 10 & 1 & 1 & 370 & DOE \\
\hline Equipment Aging & 5 & 1 & 10 & 1 & 1 & 1 & 286 & \\
\hline $\begin{array}{l}\text { Transfer Distance } \\
\text { into Roller }\end{array}$ & 10 & 5 & 1 & 1 & 1 & 5 & 278 & \\
\hline
\end{tabular}


Fig. 5 Non-quantitative example of risk assessment multivariate cause and effect relationship

\section{Demonstrated Multivariate Cause and Effect Relationships}

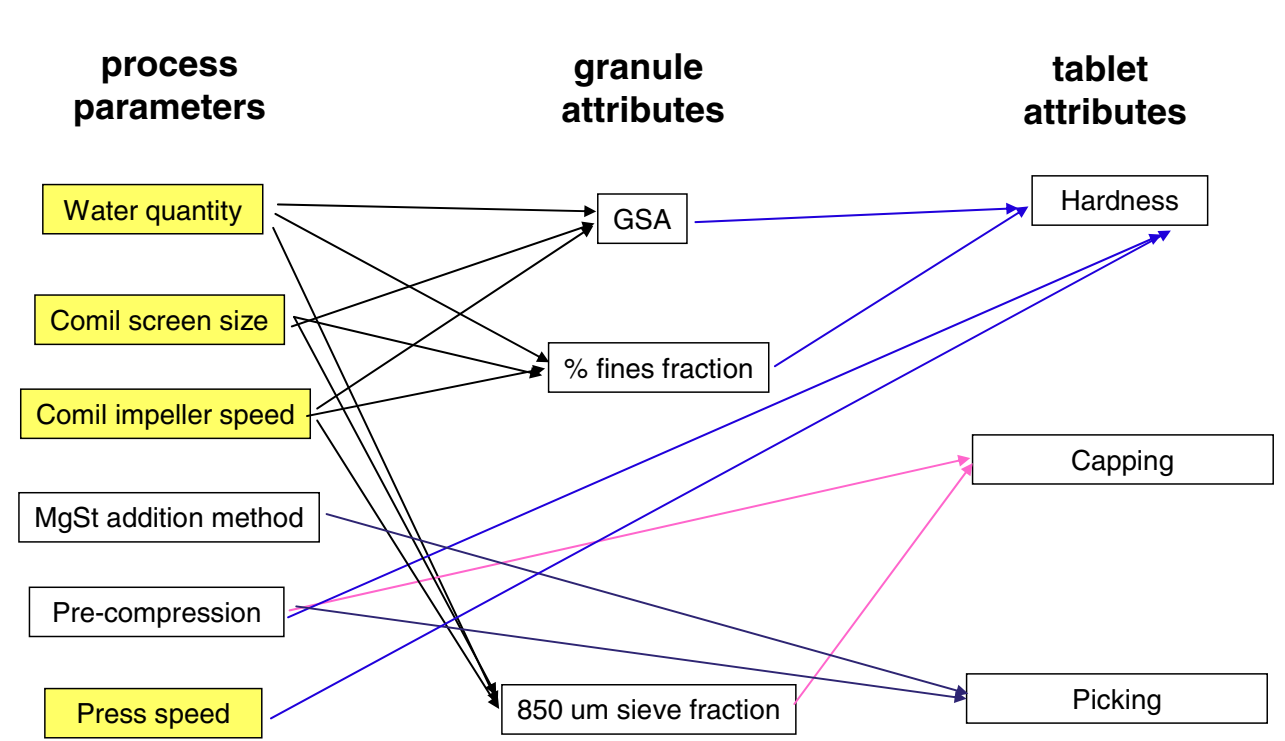

ities, and to engineer the process to drive the desired outcome. Examples are given later.

Axiom \#2 If process performance is found to be scale sensitive, the sensitivity should if possible be designed out of the process. Otherwise, conduct experiments to understand the parameters that need to be controlled in order to assure process performance. The ties to physical significance may not be as strong as mechanistic understanding, but causal relationships can be drawn.

These are usually the process elements for which scalability is improved by changing reagent addition order, or addition rate, or stoichiometry. Many of these decisions are informed by aspects that are understood or judged to be important based on a level of knowledge from Axiom \#1, but can also be informed by scale up correlations, rules-of-thumb, or definition of pseudo-kinetic order (for chemical reactions).

An example of this is mixing in chemical reactions. Some systems are sensitive to locally high reagent concentrations (as might occur when in-vessel blending is insufficient), while others are more controlled by micromixing. Blending and micromixing are known to not respond to increasing scale in the same way. In this case, specific studies would be carried out to determine which factor of interest dominates the process and use that to govern scale up.

Axiom \#3 When considering interactions of multiple variables that cannot be modelled simply, perform empirical tests (e.g., Design of Experiments - DOE) to map out appropriate operating ranges.
This approach is common in biologics and formulation, since much of the underlying science has not progressed as far as it has for synthetic molecules. It is also useful in understanding how typical fluctuations around mean input values (e.g., starting materials) can influence the final product.

Axiom \#4 Data is not knowledge, unless context is also provided. The traditional regulatory model has been to provide the data showing that the process has appropriate controls established. In a Design Space the underlying context would be necessary to show how the data was coupled with other elements, and the assumptions made.

Axiom \#5 Design Space is not necessarily the same as Proven Acceptable Range (PAR).

A combination of PARs does not add up to a Design Space. PARs based on univariate experimentation can, however, provide some knowledge about the process. When PARs are based on a multivariate assessment of the appropriate process parameters, they are a subset of design space.

\section{Presentation of the Design Space}

The presentation of Design Space is highly important in a regulated industry where trust and transparency over a product's life cycle are essential. While the Design Space Task Team has spent the majority of its effort on developing and documenting the scientific principles and approach, some 


\section{The Drug Product Design Space}

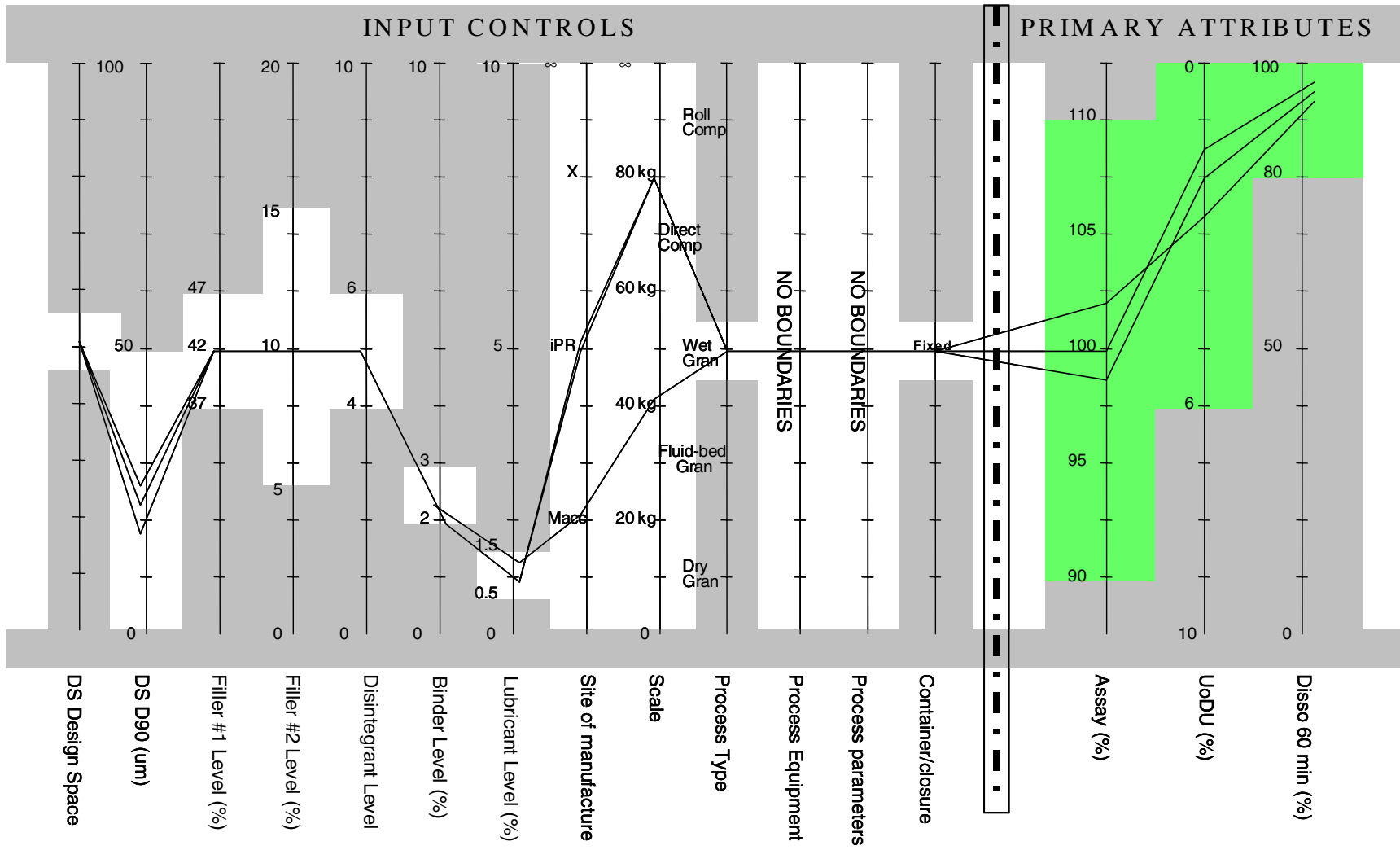

Fig. 6 Visualization of drug product design space

"common sense" considerations on presentation are given here.

The presentation of the Design Space should start with a brief guide that explains the process that was used during development to assess criticality, define the Design Space, and identify acceptable Control Strategies. This serves two purposes. Firstly, since there will be a wide range of approaches to the analysis and output, it is necessary to

\section{Case Study \#1}

\section{Control chirality by controlling process conditions}

\section{$\checkmark$ Modeling from first principles}

- PAT used to understand the reaction mechanism

$\checkmark$ Chirality is set via asymmetric hydrogenation

- Enantiomeric excess (ee) $\sim 95 \%$ in reaction stream

$\checkmark$ Enantio-selectivity decreases with increasing reaction temperature

- Temperature is critical process parameter (CPP), Max. $65^{\circ} \mathrm{C}$

- Balance kinetics and selectivity by operating at $50^{\circ} \mathrm{C}$

Fig. 7 Case study: control of process conditions

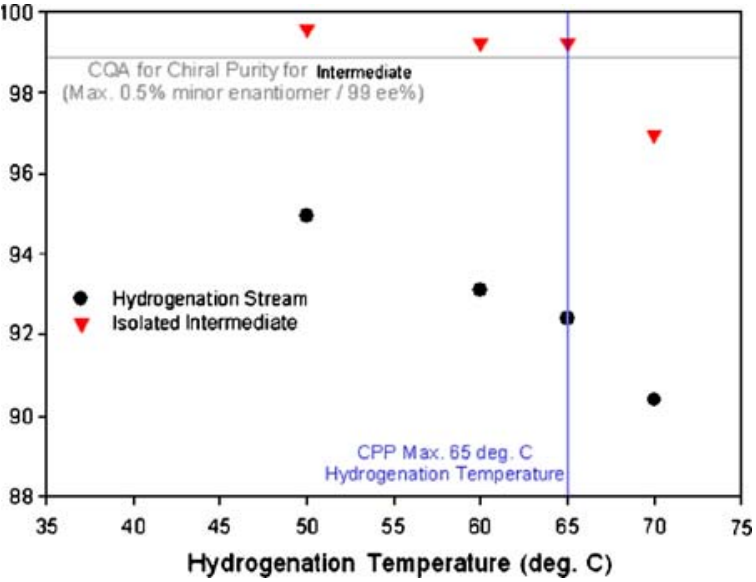




\section{Case Study \#2: Control of Drying}

Drug substance isolated in monohydrate form from isopropanol (IPA) and water

- Monohydrate form is stable under drying conditions

- Conditions thermodynamically favor removal of IPA

- Azeotrope of $88 \%$ IPA at $40^{\circ} \mathrm{C}$

> Drying temp of $40^{\circ} \mathrm{C}$ ensures maintenance of monohydrate

KF titration confirms
monohydrate and
removal of IPA
without iPA solvent
assay

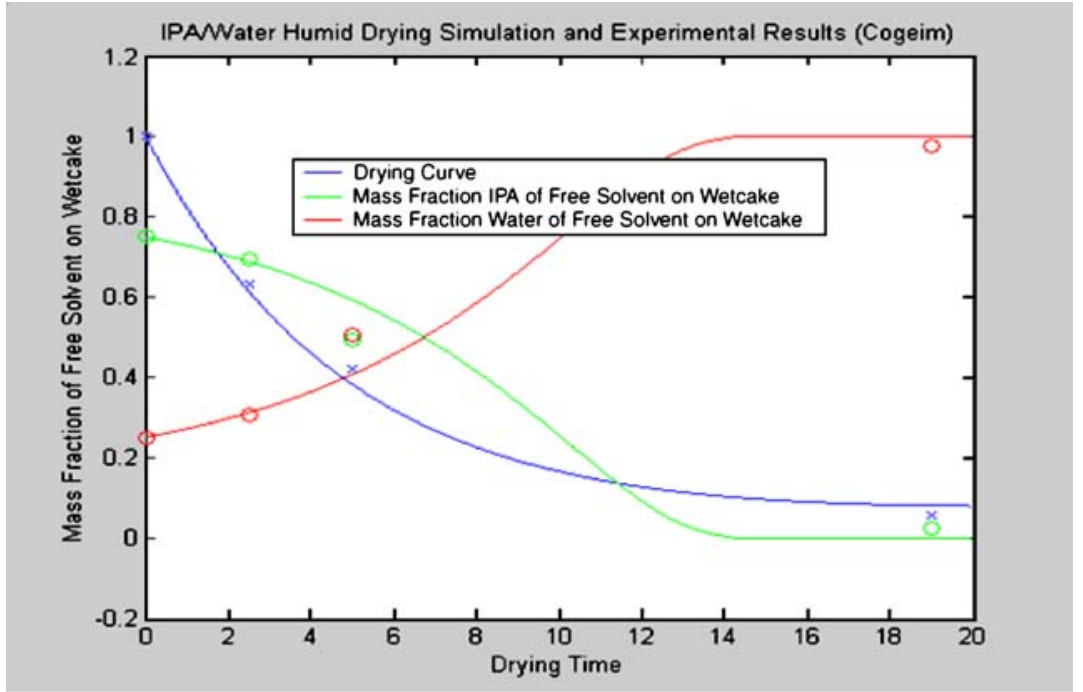

Fig. 8 Case study: control of drying

describe the overall planned process. Secondly, it is necessary for the historical record of what was done, and to provide the foundation for continuous improvement.

The goal is to define causality in sufficient detail, without excessive extraneous data or effort. The intent is to present a summary that allows easy understanding of the key elements. Technical presentation of every raw data point generated would obscure the logic that connects design considerations and design constraints.

Terms used should be clearly defined, especially any that have not been previously published or differ from widely accepted definitions (such as those listed in ICH guidance documents).

The process used for risk assessment should be described, including iterations where appropriate. This includes the identification of Critical Quality Attributes and their functional relationships with process parameters and other quality attributes that may impact them. This may include a discussion of how prior knowledge, experience and scientific literature were used during the initial risk assessments.

The information should be filtered to ensure that the data, analysis and presentation are relevant to the process discussed in the filing. Where information comes from prior knowledge or literature sources, sufficient context should be provided to allow the reviewer to see the bridge between prior knowledge and the current application. Relevance filtering also will prevent a significant level of non critical and extraneous information.

The link between risk assessments and the generation of experimental plans and models used to identify the Critical Process Parameters and their acceptable operating ranges should also be discussed. This may include statements regarding how the development process evolved to obtain additional knowledge and information to obtain further clarity on the criticality of attributes and parameters through process optimization.

\section{Design Space for Drying Graphical Description}

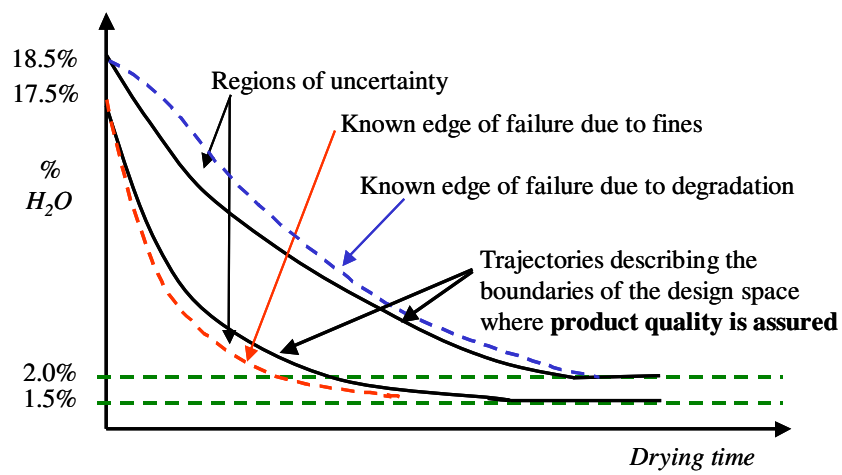

Fig. 9 Case study: design space for drying 
Fig. 10 Case study: design space for drying

\section{Experimental Design for Gerteis Study}

\section{D-Optimal Design}

- Process Parameters

Roll force

Gap width

Granulating sieve size

Granulator speed

\section{- Quality Attributes}

- Granulation particle size

- Sieve cut uniformity

- Blend potency \& uniformity

- Tablet potency \& uniformity

- Hardness at $7 \mathrm{kP}$ compress. force

- Friability at $7 \mathrm{kP}$ compression force

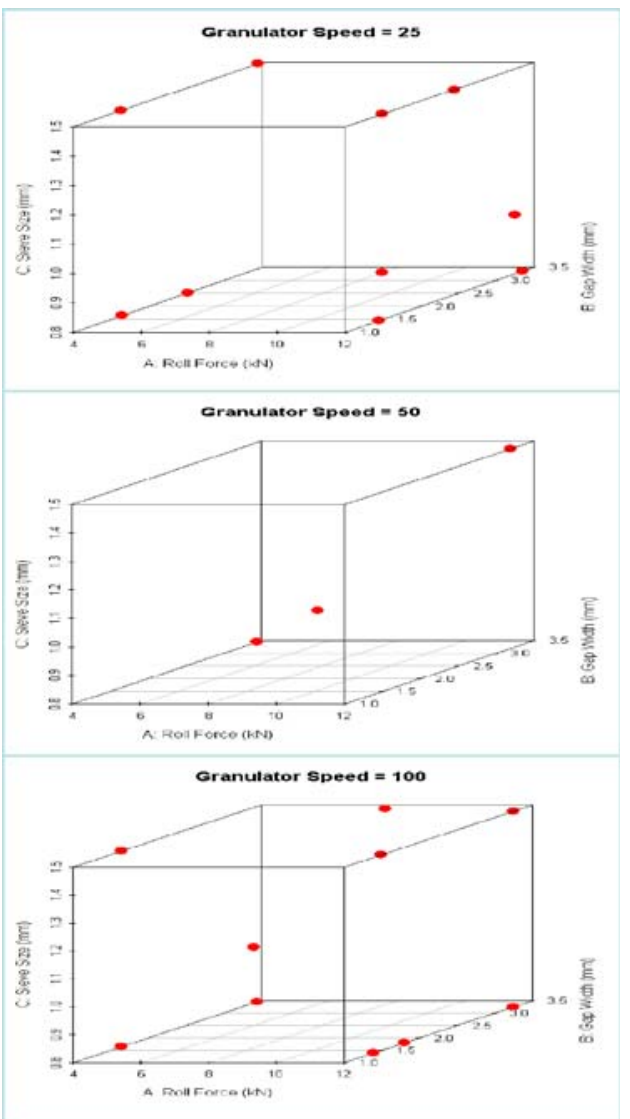

The Design Space Task Team has considered different approaches to summarizing process knowledge in a pictorial fashion. Columns containing summaries of the knowledge obtained for each unit operation may be combined into a single diagram that links information across the entire manufacturing process. Multivariate analysis, modelling and prior knowledge may all be used to prepare the information presented.

Further information on these approaches may be found in PQLI Key Topics - Criticality, Design Space and Control Strategy [2] in this Volume of Journal of Pharmaceutical Innovation.

An alternative presentation of Design Space that allows easy visualization of process is shown in Fig. 6.

\section{Case Studies}

\section{Examples from First Principles Modelling}

The first case shows a molecule in which the final chemical step was an asymmetric hydrogenation that controlled the chirality of the product. A first principle model was developed that enabled the optimization of kinetics, cycle time and selectivity of the reaction (See Fig. 7).
The second case relates to a thermodynamic model for residual solvent from drying. The model enabled the regulatory test for residual solvent to be removed from the drug substance process, relying instead on Karl Fischer (KF) titration to measure residual water. It is not thermodynamically possible under the drying conditions to have a KF meeting the drying specification with concurrent presence of a meaningful level of ispropanol (IPA). This enabled the optimization of drying cycle time and still maintained the robust production of the desired crystal form and morphology (See Fig. 8).

An alternative depiction of design space, for a different drying case study, is shown in Fig. 9. This example is based on experimentation instead of thermodynamic modelling.

\section{Examples of Empirical Models}

This example shows the use of Design of Experiment (DOE) to derive Design Space is shown in Fig. 10. Compiling the data from the experiments into a contour plot format gives the impact of compression variables on tablet properties (See Fig. 11). 
Fig. 11 Case study: impact of compression variables

\section{Example of Overlay Plot to Identify Impact of Compression Variables on Tablet Properties}

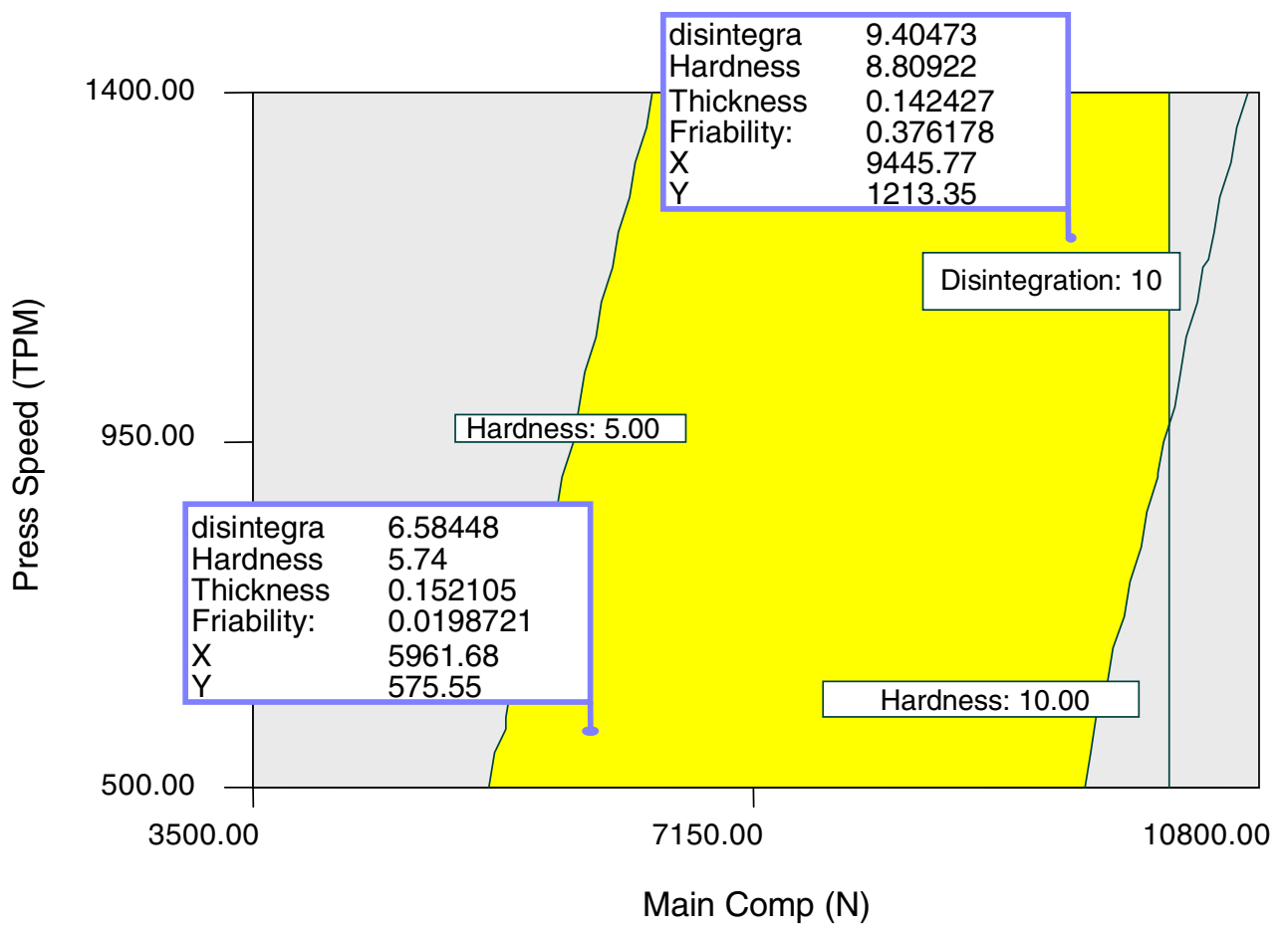

\section{Future Work and Next Steps}

Integrated examples demonstrating practical application of Design Space, criticality, and Control Strategy are necessary to further refine the concept. The following are suggested as fruitful areas for specific further study and clarification:

- Links between risk and criticality and resultant Design Space and Control Strategy. As presented here, risk management and criticality assessment form the initial steps of the Design Space definition process

- The role of scale and equipment in determining Design Space and Control Strategy

- Changes to Design Space. Since new knowledge or risks will emerge throughout the product lifecycle, this contributes to continuous improvement

- Application of Design Space principles and concepts to biologics and legacy products

- The extent to which Design Space determination includes analysis of critical and non-critical aspects, and what is eventually regulated

- The role of prior knowledge, including literature and internal technical developments captured in report form or as part of prior submissions. Prior knowledge involves many disciplines including biopharmacuetics, material classification, kinetics, thermodynamics, and experimental design techniques.

\section{References}

1. International Conference on Harmonisation of Technical Requirements for Registration of Pharmaceuticals for Human Use, ICH Harmonised Tripartite Guideline, Pharmaceutical Development Q8, Step 4, 10-November-2005.

2. PQLI Key Topics - Criticality, Design Space and Control Strategy DOI 10.1007/s12247-008-9032-4.

3. International Conference on Harmonisation of Technical Requirements for Registration of Pharmaceuticals for Human Use, ICH Harmonised Tripartite Guideline, Quality Risk Management Q9, Step 4, 9-November-2005.

4. International Conference on Harmonisation of Technical Requirements for Registration of Pharmaceuticals for Human Use, ICH Harmonised Tripartite Guideline, Pharmaceutical Quality System Q10, Step 2, 9-May-2007.

5. Procedures for Performing a Failure Mode, Effects and Criticality Analysis, United States Military Procedure MIL-P-1629, 9-November-1949.

6. EMEA PAT Team Position Paper, http://www.emea.europa.eu/ Inspections/docs/PATGuidance.pdf.

7. Criticality in PQLI DOI 10.1007/s12247-008-9033-3.

If you would like to provide comments to the authors regarding this paper, email feedback@ispe.org and include "PQLI Design Space” in the subject line. 